\title{
Construction of minimum-norm fixed points of pseudocontractions in Hilbert spaces
}

\author{
Yonghong Yao', Giuseppe Marino ${ }^{2}$, Hong-Kun $\mathrm{Xu}^{3}$ and Yeong-Cheng Liou ${ }^{4,5^{*}}$
}

"Correspondence:
simplex_liou@hotmail.com
${ }^{4}$ Department of Information
Management, Cheng Shiu
University, Kaohsiung, 833, Taiwan
${ }^{5}$ Center for General Education,
Kaohsiung Medical University,
Kaohsiung, 807, Taiwan
Full list of author information is
available at the end of the article

available at the end of the article

\begin{abstract}
An iterative algorithm is introduced for the construction of the minimum-norm fixed point of a pseudocontraction on a Hilbert space. The algorithm is proved to be strongly convergent.

MSC: $47 \mathrm{H} 05 ; 47 \mathrm{H} 10 ; 47 \mathrm{H} 17$

Keywords: fixed point; minimum-norm; pseudocontraction; nonexpansive mapping; projection
\end{abstract}

\section{Introduction}

Construction of fixed points of nonlinear mappings is a classical and active area of nonlinear functional analysis due to the fact that many nonlinear problems can be reformulated as fixed point equations of nonlinear mappings. The research of this area dates back to Picard's and Banach's time. As a matter of fact, the well-known Banach contraction principle states that the Picard iterates $\left\{T^{n} x\right\}$ converge to the unique fixed point of $T$ whenever $T$ is a contraction of a complete metric space. However, if $T$ is not a contraction (nonexpansive, say), then the Picard iterates $\left\{T^{n} x\right\}$ fail, in general, to converge; hence, other iterative methods are needed. In 1953, Mann [1] introduced the now called Mann's iterative method which generates a sequence $\left\{x_{n}\right\}$ via the averaged algorithm

$$
x_{n+1}=\left(1-\alpha_{n}\right) x_{n}+\alpha_{n} T x_{n}, \quad n \geq 0,
$$

where $\left\{\alpha_{n}\right\}$ is a sequence in the unit interval [0,1],T is a self-mapping of a closed convex subset $C$ of a Hilbert space $H$, and the initial guess $x_{0}$ is an arbitrary (but fixed) point of $C$.

Mann's algorithm (1.1) has extensively been studied [2-7], and in particular, it is known that if $T$ is nonexpansive (i.e., $\|T x-T y\| \leq\|x-y\|$ for all $x, y \in C$ ) and if $T$ has a fixed point, then the sequence $\left\{x_{n}\right\}$ generated by Mann's algorithm (1.1) converges weakly to a fixed point of $T$ provided the sequence $\left\{\alpha_{n}\right\}$ satisfies the condition

$$
\sum_{n=1}^{\infty} \alpha_{n}\left(1-\alpha_{n}\right)=\infty
$$

This algorithm, however, does not converge in the strong topology in general (see [8, Corollary 5.2]).

O2014 Yao et al.; licensee Springer. This is an Open Access article distributed under the terms of the Creative Commons Attribution License (http://creativecommons.org/licenses/by/2.0), which permits unrestricted use, distribution, and reproduction in any medium, provided the original work is properly cited. 
Browder and Petryshyn [9] studied weak convergence of Mann's algorithm (1.1) for the class of strict pseudocontractions (in the case of constant stepsizes $\alpha_{n}=\alpha$ for all $n$; see [10] for the general case of variable stepsizes). However, Mann's algorithm fails to converge for Lipschitzian pseudocontractions (see the counterexample of Chidume and Mutangadura [11]). It is therefore an interesting question of inventing iterative algorithms which generate a sequence converging in the norm topology to a fixed point of a Lipschitzian pseudocontraction (if any). The interest of pseudocontractions lies in their connection with monotone operators; namely, $T$ is a pseudocontraction if and only if the complement $I-T$ is a monotone operator.

We also notice that it is quite usual to seek a particular solution of a given nonlinear problem, in particular, the minimum-norm solution. For instance, given a closed convex subset $C$ of a Hilbert space $H_{1}$ and a bounded linear operator $A: H_{1} \rightarrow H_{2}$, where $H_{2}$ is another Hilbert space. The $C$-constrained pseudoinverse of $A, A_{C}^{\dagger}$ is then defined as the minimum-norm solution of the constrained minimization problem

$$
A_{C}^{\dagger}(b):=\arg \min _{x \in C}\|A x-b\|
$$

which is equivalent to the fixed point problem

$$
x=P_{C}\left(x-\lambda A^{*}(A x-b)\right),
$$

where $P_{C}$ is the metric projection from $H_{1}$ onto $C, A^{*}$ is the adjoint of $A, \lambda>0$ is a constant, and $b \in H_{2}$ is such that $P_{\overline{A(C)}}(b) \in A(C)$.

It is therefore an interesting problem to invent iterative algorithms that can generate sequences which converge strongly to the minimum-norm solution of a given fixed point problem. The purpose of this paper is to solve such a problem for pseudocontractions. More precisely, we shall introduce an iterative algorithm for the construction of fixed points of Lipschitzian pseudocontractions and prove that our algorithm (see (3.1) in Section 3) converges in the strong topology to the minimum-norm fixed point of the mapping.

For the existing literature on iterative methods for pseudocontractions, the reader can consult [10, 12-26]; for finding minimum-norm solutions of nonlinear fixed point and variational inequality problems, see [27-29]; and for related iterative methods for nonexpansive mappings, see $[2,3,30,31]$ and the references therein.

\section{Preliminaries}

Let $H$ be a real Hilbert space with the inner product $\langle\cdot, \cdot\rangle$ and the norm $\|\cdot\|$, respectively. Let $C$ be a nonempty closed convex subset of $H$. The class of nonlinear mappings which we will study is the class of pseudocontractions. Recall that a mapping $T: C \rightarrow C$ is a pseudocontraction if it satisfies the property

$$
\langle T x-T y, x-y\rangle \leq\|x-y\|^{2}, \quad \forall x, y \in C .
$$

It is not hard to find that $T$ is a pseudocontraction if and only if $T$ satisfies one of the following two equivalent properties:

(a) $\|T x-T y\|^{2} \leq\|x-y\|^{2}+\|(I-T) x-(I-T) y\|^{2}$ for all $x, y \in C$; or

(b) $I-T$ is monotone on $C$ : $\langle x-y,(I-T) x-(I-T) y\rangle \geq 0$ for all $x, y \in C$. 
Recall that a mapping $T: C \rightarrow C$ is nonexpansive if

$$
\|T x-T y\| \leq\|x-y\|, \quad \forall x, y \in C .
$$

It is immediately clear that nonexpansive mappings are pseudocontractions.

Recall also that the nearest point (or metric) projection from $H$ onto $C$ is defined as follows: For each point $x \in H, P_{C} x$ is the unique point in $C$ with the property

$$
\left\|x-P_{C} x\right\| \leq\|x-y\|, \quad y \in C .
$$

Note that $P_{C}$ is characterized by the inequality

$$
P_{C} x \in C, \quad\left\langle x-P_{C} x, y-P_{C} x\right\rangle \leq 0, \quad y \in C .
$$

Consequently, $P_{C}$ is nonexpansive.

In the sequel we shall use the following notations:

- Fix $(S)$ stands for the set of fixed points of $S$;

- $x_{n} \rightarrow x$ stands for the weak convergence of $\left(x_{n}\right)$ to $x$;

- $x_{n} \rightarrow x$ stands for the strong convergence of $\left(x_{n}\right)$ to $x$.

Below is the so-called demiclosedness principle for nonexpansive mappings.

Lemma 2.1 (cf. [32]) Let $C$ be a nonempty closed convex subset of a real Hilbert space $H$, and let $S: C \rightarrow C$ be a nonexpansive mapping with fixed points. If $\left\{x_{n}\right\}$ is a sequence in $C$ such that $x_{n} \rightarrow x^{*}$ and $(I-S) x_{n} \rightarrow y$, then $(I-S) x^{*}=y$.

We also need the following lemma whose proof can be found in literature (cf. [33]).

Lemma 2.2 Let $C$ be a nonempty closed convex subset of a real Hilbert space H. Assume that a mapping $F: C \rightarrow H$ is monotone and weakly continuous along segments (i.e., $F(x+t y) \rightarrow F(x)$ weakly as $t \rightarrow 0$, whenever $x+t y \in C$ for $x, y \in C)$. Then the variational inequality

$$
x^{*} \in C, \quad\left\langle F x^{*}, x-x^{*}\right\rangle \geq 0, \quad \forall x \in C
$$

is equivalent to the dual variational inequality

$$
x^{*} \in C, \quad\left\langle F x, x-x^{*}\right\rangle \geq 0, \quad \forall x \in C .
$$

Finally, we state the following elementary result on convergence of real sequences.

Lemma 2.3 ([30]) Let $\left\{a_{n}\right\}$ be a sequence of nonnegative real numbers satisfying

$$
a_{n+1} \leq\left(1-\gamma_{n}\right) a_{n}+\gamma_{n} \sigma_{n}, \quad n \geq 0,
$$

where $\left\{\gamma_{n}\right\} \subset(0,1)$ and $\left\{\sigma_{n}\right\}$ satisfy

(i) $\sum_{n=0}^{\infty} \gamma_{n}=\infty$;

(ii) either $\lim \sup _{n \rightarrow \infty} \sigma_{n} \leq 0$ or $\sum_{n=0}^{\infty}\left|\gamma_{n} \sigma_{n}\right|<\infty$.

Then $\left\{a_{n}\right\}$ converges to 0 . 


\section{An iterative algorithm and its convergence}

Throughout this section we assume that $C$ is a nonempty closed subset of a real Hilbert space $H$ and $T: C \rightarrow C$ is a pseudocontraction with a nonempty fixed point set $\operatorname{Fix}(T)$. The aim of this section is to introduce an iterative method for finding the minimum-norm fixed point of $T$. Towards this, we select two sequences of real numbers, $\left\{\alpha_{n}\right\}$ and $\left\{\beta_{n}\right\}$ in the interval $(0,1)$ such that

$$
\alpha_{n}+\beta_{n}<1
$$

for all $n$. We also take an arbitrary initial guess $x_{0} \in C$. We then define an iterative algorithm which generates a sequence $\left\{x_{n}\right\}$ via the following recursion:

$$
x_{n+1}=P_{C}\left[\left(1-\alpha_{n}-\beta_{n}\right) x_{n}+\beta_{n} T x_{n}\right], \quad n \geq 0 \text {. }
$$

We shall prove that this sequence strongly converges to the minimum-norm fixed point of $T$ provided $\left\{\alpha_{n}\right\}$ and $\left\{\beta_{n}\right\}$ satisfy certain conditions. To this end, we need the following lemma.

Lemma 3.1 Let $f: C \rightarrow H$ be a contraction with coefficient $\rho \in(0,1)$. Let $S: C \rightarrow C$ be a nonexpansive mapping with $\operatorname{Fix}(S) \neq \emptyset$. For each $t \in(0,1)$, let $x_{t}$ be defined as the unique solution of the fixed point equation

$$
x_{t}=S P_{C}\left[t f\left(x_{t}\right)+(1-t) x_{t}\right]
$$

Then, as $t \rightarrow 0^{+}$, the net $\left\{x_{t}\right\}$ converges strongly to a point $x^{*} \in \operatorname{Fix}(S)$ which solves the following variational inequality:

$$
x^{*} \in \operatorname{Fix}(S), \quad\left\langle(I-f) x^{*}, x-x^{*}\right\rangle \geq 0, \quad x \in \operatorname{Fix}(S) .
$$

In particular, if we take $f=0$, then the net $\left\{x_{t}\right\}$ defined via the fixed point equation

$$
x_{t}=S P_{C}\left[(1-t) x_{t}\right]
$$

converges in norm, as $t \rightarrow 0^{+}$, to the minimum-norm fixed point of $S$.

Proof First observe that, for each $t \in(0,1), x_{t}$ is well defined. Indeed, if we define a mapping $S_{t}: C \rightarrow C$ by

$$
S_{t} x=S P_{C}[t f(x)+(1-t) x], \quad x \in C .
$$

For $x, y \in C$, we have

$$
\begin{aligned}
\left\|S_{t} x-S_{t} y\right\| & =\left\|S P_{C}[t f(x)+(1-t) x]-S P_{C}[t f(y)+(1-t) y]\right\| \\
& \leq t\|f(x)-f(y)\|+(1-t)\|x-y\| \\
& \leq[1-(1-\rho) t]\|x-y\|,
\end{aligned}
$$


which implies that $S_{t}$ is a self-contraction of $C$. Hence $S_{t}$ has a unique fixed point $x_{t} \in C$ which is the unique solution of fixed point equation (3.3).

Next we prove that $\left\{x_{t}\right\}$ is bounded. Take $u \in \operatorname{Fix}(S)$. From (3.3) we have

$$
\begin{aligned}
\left\|x_{t}-u\right\| & =\left\|S P_{C}\left[t f\left(x_{t}\right)+(1-t) x_{t}\right]-S P_{C} u\right\| \\
& \leq t\left\|f\left(x_{t}\right)-f(u)\right\|+t\|f(u)-u\|+(1-t)\left\|x_{t}-u\right\| \\
& \leq[1-(1-\rho) t]\left\|x_{t}-u\right\|+t\|f(u)-u\|,
\end{aligned}
$$

that is,

$$
\left\|x_{t}-u\right\| \leq \frac{\|f(u)-u\|}{1-\rho} .
$$

Hence, $\left\{x_{t}\right\}$ is bounded and so is $\left\{f\left(x_{t}\right)\right\}$.

From (3.3) we have

$$
\begin{aligned}
\left\|x_{t}-S x_{t}\right\| & =\left\|S P_{C}\left[t f\left(x_{t}\right)+(1-t) x_{t}\right]-S P_{C} x_{t}\right\| \\
& \leq t\left\|f\left(x_{t}\right)-x_{t}\right\| \rightarrow 0 \quad \text { as } t \rightarrow 0^{+} .
\end{aligned}
$$

Next we show that $\left\{x_{t}\right\}$ is relatively norm-compact as $t \rightarrow 0^{+}$, i.e., we show that from any sequence in $\left\{x_{t}\right\}$, a convergent subsequence can be extracted. Let $\left\{t_{n}\right\} \subset(0,1)$ be a sequence such that $t_{n} \rightarrow 0^{+}$as $n \rightarrow \infty$. Put $x_{n}:=x_{t_{n}}$. From (3.5) we have

$$
\left\|x_{n}-S x_{n}\right\| \rightarrow 0
$$

Again from (3.3) we get

$$
\begin{aligned}
\left\|x_{t}-u\right\|^{2}= & \left\|S P_{C}\left[t f\left(x_{t}\right)+(1-t) x_{t}\right]-S P_{C} u\right\|^{2} \\
\leq & \left\|x_{t}-u+t\left(f\left(x_{t}\right)-x_{t}\right)\right\|^{2} \\
= & \left\|x_{t}-u\right\|^{2}+2 t\left\langle f\left(x_{t}\right)-x_{t}, x_{t}-u\right\rangle+t^{2}\left\|f\left(x_{t}\right)-x_{t}\right\|^{2} \\
= & \left\|x_{t}-u\right\|^{2}+2 t\left\langle f\left(x_{t}\right)-f(u), x_{t}-u\right\rangle+2 t\left\langle f(u)-u, x_{t}-u\right\rangle \\
& +2 t\left\langle u-x_{t}, x_{t}-u\right\rangle+t^{2}\left\|f\left(x_{t}\right)-x_{t}\right\|^{2} \\
\leq & {[1-2(1-\rho) t]\left\|x_{t}-u\right\|^{2}+2 t\left|f(u)-u, x_{t}-u\right\rangle+t^{2}\left\|f\left(x_{t}\right)-x_{t}\right\|^{2} . }
\end{aligned}
$$

It turns out that

$$
\left\|x_{t}-u\right\|^{2} \leq \frac{1}{1-\rho}\left\langle f(u)-u, x_{t}-u\right\rangle+t M
$$

where $M>0$ is a constant such that

$$
M>\frac{1}{2(1-\rho)} \sup \left\{\left\|f\left(x_{t}\right)-x_{t}\right\|^{2}: t \in(0,1)\right\} .
$$

In particular, we get from (3.7)

$$
\left\|x_{n}-u\right\|^{2} \leq \frac{1}{1-\rho}\left\langle f(u)-u, x_{n}-u\right\rangle+t_{n} M, \quad u \in \operatorname{Fix}(S) .
$$


Since $\left\{x_{n}\right\}$ is bounded, without loss of generality, we may assume that $\left\{x_{n}\right\}$ converges weakly to a point $x^{*} \in C$. Noticing (3.6) we can use Lemma 2.1 to get $x^{*} \in \operatorname{Fix}(S)$. Therefore we can substitute $x^{*}$ for $u$ in (3.8) to get

$$
\left\|x_{n}-x^{*}\right\|^{2} \leq \frac{1}{1-\rho}\left\langle f\left(x^{*}\right)-x^{*}, x_{n}-x^{*}\right\rangle+t_{n} M
$$

However, $x_{n} \rightarrow x^{*}$. This together with (3.9) guarantees that $x_{n} \rightarrow x^{*}$. The net $\left\{x_{t}\right\}$ is therefore relatively compact, as $t \rightarrow 0^{+}$, in the norm topology.

Now we return to (3.8) and take the limit as $n \rightarrow \infty$ to get

$$
\left\|x^{*}-u\right\|^{2} \leq \frac{1}{1-\rho}\left\langle f(u)-u, x^{*}-u\right\rangle, \quad u \in \operatorname{Fix}(S) .
$$

In particular, $x^{*}$ solves the following variational inequality:

$$
x^{*} \in \operatorname{Fix}(S), \quad\left\langle(I-f) u, u-x^{*}\right\rangle \geq 0, \quad u \in \operatorname{Fix}(S) .
$$

By Lemma 2.2, we see that $x^{*}$ solves the variational inequality

$$
x^{*} \in \operatorname{Fix}(S), \quad\left\langle(I-f) x^{*}, u-x^{*}\right\rangle \geq 0, \quad u \in \operatorname{Fix}(S) .
$$

Therefore, $x^{*}=\left(P_{\mathrm{Fix}(S)} f\right) x^{*}$. That is, $x^{*}$ is the unique fixed point in Fix $(S)$ of the contraction $P_{\mathrm{Fix}(S)} f$. Clearly this is sufficient to conclude that the entire net $\left\{x_{t}\right\}$ converges in norm to $x^{*}$ as $t \rightarrow 0^{+}$.

Finally, if we take $f=0$, then variational inequality (3.10) is reduced to

$$
0 \leq\left\langle x^{*}, u-x^{*}\right\rangle, \quad u \in \operatorname{Fix}(S)
$$

Equivalently,

$$
\left\|x^{*}\right\|^{2} \leq\left\langle x^{*}, u\right\rangle, \quad u \in \operatorname{Fix}(S)
$$

This clearly implies that

$$
\left\|x^{*}\right\| \leq\|u\|, \quad u \in \operatorname{Fix}(S)
$$

Therefore, $x^{*}$ is the minimum-norm fixed point of $S$. This completes the proof.

We are now in a position to prove the strong convergence of algorithm (3.2).

Theorem 3.2 Let $C$ be a nonempty closed convex subset of a real Hilbert space $H$, and let $T: C \rightarrow C$ be L-Lipschitzian and pseudocontractive with $\operatorname{Fix}(T) \neq \emptyset$. Suppose that the following conditions are satisfied:

(i) $\lim _{n \rightarrow \infty} \alpha_{n}=0$ and $\sum_{n=0}^{\infty} \alpha_{n}=\infty$;

(ii) $\lim _{n \rightarrow \infty} \frac{\alpha_{n}}{\beta_{n}}=\lim _{n \rightarrow \infty} \frac{\beta_{n}^{2}}{\alpha_{n}}=0$;

(iii) $\lim _{n \rightarrow \infty} \frac{\alpha_{n} \beta_{n-1}-\alpha_{n-1} \beta_{n}}{\alpha_{n}^{2} \beta_{n-1}}=0$. 
Then the sequence $\left\{x_{n}\right\}$ generated by algorithm (3.2) converges strongly to the minimumnorm fixed point of $T$.

Proof First we prove that the sequence $\left\{x_{n}\right\}$ is bounded. We will show this fact by induction. According to conditions (i) and (ii), there exists a sufficiently large positive integer $m$ such that

$$
1-2(L+1)(L+2)\left(\alpha_{n}+2 \beta_{n}+\frac{\beta_{n}^{2}}{\alpha_{n}}\right)>0, \quad n \geq m .
$$

Fix $p \in \operatorname{Fix}(T)$ and take a constant $M_{1}>0$ such that

$$
\max \left\{\left\|x_{0}-p\right\|,\left\|x_{1}-p\right\|, \ldots,\left\|x_{m}-p\right\|, 2\|p\|\right\} \leq M_{1}
$$

Next, we show that $\left\|x_{m+1}-p\right\| \leq M_{1}$.

Set

$$
y_{m}=\left(1-\alpha_{m}-\beta_{m}\right) x_{m}+\beta_{m} T x_{m} ; \quad \text { thus } x_{m+1}=P_{C}\left[y_{m}\right]
$$

Then, by using property (2.2) of the metric projection, we have

$$
\left\langle x_{m+1}-y_{m}, x_{m+1}-p\right\rangle \leq 0 \text {. }
$$

By the fact that $I-T$ is monotone, we have

$$
\left\langle(I-T) x_{m+1}-(I-T) p, x_{m+1}-p\right\rangle \geq 0 .
$$

From (3.2), (3.13) and (3.14), we obtain

$$
\begin{aligned}
\left\|x_{m+1}-p\right\|^{2}= & \left\langle x_{m+1}-p, x_{m+1}-p\right\rangle \\
= & \left\langle x_{m+1}-y_{m}, x_{m+1}-p\right\rangle+\left\langle y_{m}-p, x_{m+1}-p\right\rangle \\
\leq & \left\langle y_{m}-p, x_{m+1}-p\right\rangle \\
= & \left\langle x_{m}-p, x_{m+1}-p\right\rangle-\alpha_{m}\left\langle x_{m}, x_{m+1}-p\right\rangle+\beta_{m}\left\langle T x_{m}-x_{m}, x_{m+1}-p\right\rangle \\
= & \left\langle x_{m}-p, x_{m+1}-p\right\rangle+\alpha_{m}\left\langle x_{m+1}-x_{m}, x_{m+1}-p\right\rangle-\alpha_{m}\left\langle p, x_{m+1}-p\right\rangle \\
& -\alpha_{m}\left\langle x_{m+1}-p, x_{m+1}-p\right\rangle+\beta_{m}\left\langle T x_{m}-T x_{m+1}, x_{m+1}-p\right\rangle \\
& +\beta_{m}\left\langle x_{m+1}-x_{m}, x_{m+1}-p\right\rangle-\beta_{m}\left\langle x_{m+1}-T x_{m+1}, x_{m+1}-p\right\rangle \\
\leq & \left\|x_{m}-p\right\|\left\|x_{m+1}-p\right\|+\alpha_{m}\left\|x_{m+1}-x_{m}\right\|\left\|x_{m+1}-p\right\| \\
& +\alpha_{m}\|p\|\left\|x_{m+1}-p\right\|-\alpha_{m}\left\|x_{m+1}-p\right\|^{2} \\
& +\beta_{m}\left(\left\|T x_{m}-T x_{m+1}\right\|+\left\|x_{m+1}-x_{m}\right\|\right)\left\|x_{m+1}-p\right\| \\
\leq & \left\|x_{m}-p\right\|\left\|x_{m+1}-p\right\|+\alpha_{m}\|p\|\left\|x_{m+1}-p\right\|-\alpha_{m}\left\|x_{m+1}-p\right\|^{2} \\
& +(L+1)\left(\alpha_{m}+\beta_{m}\right)\left\|x_{m+1}-x_{m}\right\|\left\|x_{m+1}-p\right\| .
\end{aligned}
$$


It follows that

$$
\left(1+\alpha_{m}\right)\left\|x_{m+1}-p\right\| \leq\left\|x_{m}-p\right\|+\alpha_{m}\|p\|+(L+1)\left(\alpha_{m}+\beta_{m}\right)\left\|x_{m+1}-x_{m}\right\| .
$$

By (3.2), we have

$$
\begin{aligned}
\left\|x_{m+1}-x_{m}\right\| & =\left\|P_{C}\left[\left(1-\alpha_{m}-\beta_{m}\right) x_{m}+\beta_{m} T x_{m}\right]-P_{C}\left[x_{m}\right]\right\| \\
& \leq\left\|\left(1-\alpha_{m}-\beta_{m}\right) x_{m}+\beta_{m} T x_{m}-x_{m}\right\| \\
& \leq \alpha_{m}\left(\|p\|+\left\|x_{m}-p\right\|\right)+\beta_{m}\left(\left\|T x_{m}-p\right\|+\left\|x_{m}-p\right\|\right) \\
& \leq \alpha_{m}\left(\|p\|+\left\|x_{m}-p\right\|\right)+(L+1) \beta_{m}\left\|x_{m}-p\right\| \\
& \leq(L+1)\left(\alpha_{m}+\beta_{m}\right)\left\|x_{m}-p\right\|+\alpha_{m}\|p\| \\
& \leq(L+2)\left(\alpha_{m}+\beta_{m}\right) M_{1} .
\end{aligned}
$$

Substitute (3.16) into (3.15) to obtain

$$
\begin{aligned}
\left(1+\alpha_{m}\right)\left\|x_{m+1}-p\right\| & \leq\left\|x_{m}-p\right\|+\alpha_{m}\|p\|+(L+1)(L+2)\left(\alpha_{m}+\beta_{m}\right)^{2} M_{1} \\
& \leq\left(1+\frac{1}{2} \alpha_{m}\right) M_{1}+(L+1)(L+2)\left(\alpha_{m}+\beta_{m}\right)^{2} M_{1}
\end{aligned}
$$

that is,

$$
\begin{aligned}
\left\|x_{m+1}-p\right\| & \leq\left[1-\frac{\left(\alpha_{m} / 2\right)-(L+1)(L+2)\left(\alpha_{m}+\beta_{m}\right)^{2}}{1+\alpha_{m}}\right] M_{1} \\
& =\left\{1-\frac{\left(\alpha_{m} / 2\right)\left[1-2(L+1)(L+2)\left(\alpha_{m}+2 \beta_{m}+\left(\beta_{m}^{2} / \alpha_{m}\right)\right)\right]}{1+\alpha_{m}}\right\} M_{1} \\
& \leq M_{1} .
\end{aligned}
$$

By induction, we get

$$
\left\|x_{n}-p\right\| \leq M_{1}, \quad \forall n \geq 0,
$$

which implies that $\left\{x_{n}\right\}$ is bounded and so is $\left\{T x_{n}\right\}$. Now we take a constant $M_{2}>0$ such that

$$
M_{2}=\sup _{n}\left\{\left\|x_{n}\right\| \vee\left\|T x_{n}-x_{n}\right\|\right\}
$$

[Here $a \vee b=\max \{a, b\}$ for $a, b \in \mathbb{R}$.]

Set $S=(2 I-T)^{-1}$ (i.e., $S$ is a resolvent of the monotone operator $\left.I-T\right)$. We then have that $S$ is a nonexpansive self-mapping of $C$ and $\operatorname{Fix}(S)=\operatorname{Fix}(T)$ ( $c f$. Theorem 6 of [34]).

By Lemma 3.1, we know that whenever $\left\{\gamma_{n}\right\} \subset(0,1)$ and $\gamma_{n} \rightarrow 0^{+}$, the sequence $\left\{z_{n}\right\}$ defined by

$$
z_{n}=S P_{C}\left[\left(1-\gamma_{n}\right) z_{n}\right]
$$


converges strongly to the minimum-norm fixed point $x^{*}$ of $S$ (and of $T$ as $\operatorname{Fix}(S)=\operatorname{Fix}(T)$ ). Without loss of generality, we may assume that $\left\|z_{n}\right\| \leq M_{2}$ for all $n$.

It suffices to prove that $\left\|x_{n+1}-z_{n}\right\| \rightarrow 0$ as $n \rightarrow \infty$ (for some $\gamma_{n} \rightarrow 0^{+}$). To this end, we rewrite (3.18) as

$$
(2 I-T) z_{n}=P_{C}\left[\left(1-\gamma_{n}\right) z_{n}\right], \quad n \geq 0 .
$$

By using the property of metric projection (2.2), we have

$$
\begin{aligned}
\left\langle\left(1-\gamma_{n}\right) z_{n}-\left(2 z_{n}-T z_{n}\right), x_{n+1}-\left(2 z_{n}-T z_{n}\right)\right\rangle \leq 0 \\
\quad \Rightarrow \quad\left\langle-\gamma_{n} z_{n}, x_{n+1}-z_{n}-\left(z_{n}-T z_{n}\right)\right\rangle+\left\langle T z_{n}-z_{n}, x_{n+1}-z_{n}-\left(z_{n}-T z_{n}\right)\right\rangle \leq 0 \\
\quad \Rightarrow \quad\left\langle-\gamma_{n} z_{n}+T z_{n}-z_{n}, x_{n+1}-z_{n}\right\rangle+\left\|z_{n}-T z_{n}\right\|^{2} \leq\left\langle\gamma_{n} z_{n}, T z_{n}-z_{n}\right\rangle \\
\quad \Rightarrow \quad\left\langle-\gamma_{n} z_{n}+T z_{n}-z_{n}, x_{n+1}-z_{n}\right\rangle \leq \gamma_{n}\left\|z_{n}\right\|\left\|T z_{n}-z_{n}\right\| \\
\quad \Rightarrow \quad\left\langle-z_{n}+\frac{T z_{n}-z_{n}}{\gamma_{n}}, x_{n+1}-z_{n}\right\rangle \leq\left\|z_{n}\right\|\left\|T z_{n}-z_{n}\right\| .
\end{aligned}
$$

Note that

$$
\begin{aligned}
\left\|z_{n}-T z_{n}\right\| & =\left\|P_{C}\left[\left(1-\gamma_{n}\right) z_{n}\right]-z_{n}\right\| \\
& \leq\left\|\left(1-\gamma_{n}\right) z_{n}-z_{n}\right\| \\
& =\gamma_{n}\left\|z_{n}\right\| .
\end{aligned}
$$

Hence, we get

$$
\left\langle-z_{n}+\frac{T z_{n}-z_{n}}{\gamma_{n}}, x_{n+1}-z_{n}\right\rangle \leq \gamma_{n}\left\|z_{n}\right\|^{2}
$$

From (3.18) we have

$$
\begin{aligned}
\left\|z_{n+1}-z_{n}\right\| & =\left\|S P_{C}\left[\left(1-\gamma_{n+1}\right) z_{n+1}\right]-S P_{C}\left[\left(1-\gamma_{n}\right) z_{n}\right]\right\| \\
& \leq\left\|\left(1-\gamma_{n+1}\right) z_{n+1}-\left(1-\gamma_{n}\right) z_{n}\right\| \\
& =\left\|\left(1-\gamma_{n+1}\right)\left(z_{n+1}-z_{n}\right)+\left(\gamma_{n}-\gamma_{n+1}\right) z_{n}\right\| \\
& \leq\left(1-\gamma_{n+1}\right)\left\|z_{n+1}-z_{n}\right\|+\left|\gamma_{n+1}-\gamma_{n}\right|\left\|z_{n}\right\| .
\end{aligned}
$$

It follows that

$$
\left\|z_{n+1}-z_{n}\right\| \leq \frac{\left|\gamma_{n+1}-\gamma_{n}\right|}{\gamma_{n+1}}\left\|z_{n}\right\|
$$

Set

$$
\gamma_{n}:=\frac{\alpha_{n}}{\beta_{n}}
$$


By condition (ii), $\gamma_{n} \rightarrow 0^{+}$and $\gamma_{n} \in(0,1)$ for $n$ large enough. Hence, by (3.19) and (3.20) we have

$$
\left\langle-z_{n}+\frac{\beta_{n}\left(T z_{n}-z_{n}\right)}{\alpha_{n}}, x_{n+1}-z_{n}\right\rangle \leq \frac{\alpha_{n}}{\beta_{n}}\left\|z_{n}\right\|^{2} \leq \frac{\alpha_{n}}{\beta_{n}} M_{2}^{2}
$$

and

$$
\left\|z_{n}-z_{n-1}\right\| \leq \frac{\alpha_{n} \beta_{n-1}-\alpha_{n-1} \beta_{n}}{\alpha_{n} \beta_{n-1}} M_{2}
$$

By (3.2) we have

$$
\begin{aligned}
\left\|x_{n+1}-x_{n}\right\| & =\left\|P_{C}\left[\left(1-\alpha_{n}-\beta_{n}\right) x_{n}+\beta_{n} T x_{n}\right]-P_{C} x_{n}\right\| \\
& \leq \alpha_{n}\left\|x_{n}\right\|+\beta_{n}\left\|T x_{n}-x_{n}\right\| \\
& \leq\left(\alpha_{n}+\beta_{n}\right) M_{2} .
\end{aligned}
$$

Next, we estimate $\left\|x_{n+1}-z_{n+1}\right\|$. Since $x_{n+1}=P_{C}\left[y_{n}\right],\left\langle x_{n+1}-y_{n}, x_{n+1}-z_{n}\right\rangle \leq 0$. Using (3.21) and by the fact that $T$ is $L$-Lipschitzian and pseudocontractive, we infer that

$$
\begin{aligned}
\left\|x_{n+1}-z_{n}\right\|^{2}= & \left\langle x_{n+1}-z_{n}, x_{n+1}-z_{n}\right\rangle \\
= & \left\langle x_{n+1}-y_{n}, x_{n+1}-z_{n}\right\rangle+\left\langle y_{n}-z_{n}, x_{n+1}-z_{n}\right\rangle \\
\leq & \left\langle y_{n}-z_{n}, x_{n+1}-z_{n}\right\rangle \\
= & \left\langle\left[\left(1-\alpha_{n}-\beta_{n}\right) x_{n}+\beta_{n} T x_{n}\right]-z_{n}, x_{n+1}-z_{n}\right\rangle \\
= & \left(1-\alpha_{n}-\beta_{n}\right)\left\langle x_{n}-z_{n}, x_{n+1}-z_{n}\right\rangle+\beta_{n}\left\langle T x_{n}-T x_{n+1}, x_{n+1}-z_{n}\right\rangle \\
& +\beta_{n}\left\langle T x_{n+1}-T z_{n}, x_{n+1}-z_{n}\right\rangle+\left\langle-\alpha_{n} z_{n}+\beta_{n}\left(T z_{n}-z_{n}\right), x_{n+1}-z_{n}\right\rangle,
\end{aligned}
$$

which leads to

$$
\begin{aligned}
\left\|x_{n+1}-z_{n}\right\|^{2} \leq & \left(1-\alpha_{n}-\beta_{n}\right)\left\|x_{n}-z_{n}\right\|\left\|x_{n+1}-z_{n}\right\|+\beta_{n} L\left\|x_{n}-x_{n+1}\right\|\left\|x_{n+1}-z_{n}\right\| \\
& +\beta_{n}\left\|x_{n+1}-z_{n}\right\|^{2}+\alpha_{n}\left(-z_{n}+\frac{\beta_{n}}{\alpha_{n}}\left(T z_{n}-z_{n}\right), x_{n+1}-z_{n}\right) \\
\leq & \frac{1-\alpha_{n}-\beta_{n}}{2}\left(\left\|x_{n}-z_{n}\right\|^{2}+\left\|x_{n+1}-z_{n}\right\|^{2}\right)+\frac{\beta_{n}^{2}}{2}\left\|x_{n+1}-z_{n}\right\|^{2} \\
& +\frac{L^{2}}{2}\left\|x_{n}-x_{n+1}\right\|^{2}+\beta_{n}\left\|x_{n+1}-z_{n}\right\|^{2}+\frac{\alpha_{n}^{2}}{\beta_{n}}\left\|z_{n}\right\|^{2} .
\end{aligned}
$$

It follows that, using (3.21), (3.22) and (3.23), we get

$$
\begin{aligned}
\left\|x_{n+1}-z_{n}\right\|^{2} \leq & \frac{1-\alpha_{n}-\beta_{n}}{1+\alpha_{n}-\beta_{n}}\left\|x_{n}-z_{n}\right\|^{2}+\frac{L^{2}}{1+\alpha_{n}-\beta_{n}}\left\|x_{n+1}-x_{n}\right\|^{2} \\
& +\frac{2 \alpha_{n}^{2}}{\left(1+\alpha_{n}-\beta_{n}\right) \beta_{n}}\left\|z_{n}\right\|^{2}+\frac{\beta_{n}^{2}}{1+\alpha_{n}-\beta_{n}}\left\|x_{n+1}-z_{n}\right\|^{2} \\
\leq & \left(1-\frac{2 \alpha_{n}}{1+\alpha_{n}-\beta_{n}}\right)\left\|x_{n}-z_{n}\right\|^{2}+\frac{\left(\alpha_{n}+\beta_{n}\right)^{2}}{1+\alpha_{n}-\beta_{n}} L^{2} M_{2}^{2}
\end{aligned}
$$




$$
\begin{aligned}
& +\frac{2 \alpha_{n}^{2}}{\left(1+\alpha_{n}-\beta_{n}\right) \beta_{n}} M_{2}^{2}+\frac{\beta_{n}^{2}}{1+\alpha_{n}-\beta_{n}} 4 M_{2}^{2} \\
\leq & \left(1-\frac{2 \alpha_{n}}{1+\alpha_{n}-\beta_{n}}\right)\left(\left\|x_{n}-z_{n-1}\right\|+\left\|z_{n}-z_{n-1}\right\|\right)^{2} \\
& +\left\{\frac{\left(\alpha_{n}+\beta_{n}\right)^{2}}{1+\alpha_{n}-\beta_{n}}+\frac{2 \alpha_{n}^{2}}{\left(1+\alpha_{n}-\beta_{n}\right) \beta_{n}}+\frac{\beta_{n}^{2}}{1+\alpha_{n}-\beta_{n}}\right\} M \\
\leq & \left(1-\frac{2 \alpha_{n}}{1+\alpha_{n}-\beta_{n}}\right)\left\|x_{n}-z_{n-1}\right\|^{2} \\
& +\frac{1}{1+\alpha_{n}-\beta_{n}}\left\|z_{n}-z_{n-1}\right\|\left(2\left\|x_{n}-z_{n-1}\right\|+\left\|z_{n}-z_{n-1}\right\|\right) \\
& +\left\{\frac{\left(\alpha_{n}+\beta_{n}\right)^{2}}{1+\alpha_{n}-\beta_{n}}+\frac{2 \alpha_{n}^{2}}{\left(1+\alpha_{n}-\beta_{n}\right) \beta_{n}}+\frac{\beta_{n}^{2}}{1+\alpha_{n}-\beta_{n}}\right\} M \\
\leq & \left(1-\frac{2 \alpha_{n}}{1+\alpha_{n}-\beta_{n}}\right)\left\|x_{n}-z_{n-1}\right\|^{2}+\frac{1}{1+\alpha_{n}-\beta_{n}} \frac{\alpha_{n} \beta_{n-1}-\alpha_{n-1} \beta_{n}}{\alpha_{n} \beta_{n-1}} M \\
& +\left\{\frac{\left(\alpha_{n}+\beta_{n}\right)^{2}}{1+\alpha_{n}-\beta_{n}}+\frac{2 \alpha_{n}^{2}}{\left(1+\alpha_{n}-\beta_{n}\right) \beta_{n}}+\frac{\beta_{n}^{2}}{1+\alpha_{n}-\beta_{n}}\right\} M,
\end{aligned}
$$

where the finite constant $M>0$ is given by

$$
M:=\max \left\{L^{2} M_{2}^{2}, 4 M_{2}^{2}, M_{2} \sup _{n}\left(2\left\|x_{n}-z_{n-1}\right\|+\left\|z_{n}-z_{n-1}\right\|\right)\right\} .
$$

Let

$$
\delta_{n}=\frac{2 \alpha_{n}}{1+\alpha_{n}-\beta_{n}} \approx 2 \alpha_{n} \quad(\text { as } n \rightarrow \infty)
$$

and note that by $(3.1)$ it follows that $\left\{\delta_{n}\right\} \subset(0,1)$. Moreover, set

$$
\theta_{n}=\left\{\frac{\alpha_{n} \beta_{n-1}-\alpha_{n-1} \beta_{n}}{2 \alpha_{n}^{2} \beta_{n-1}}+\frac{1}{2}\left(\alpha_{n}+2 \beta_{n}+\frac{\beta_{n}^{2}}{\alpha_{n}}\right)+\frac{\alpha_{n}}{\beta_{n}}+\frac{\beta_{n}^{2}}{2 \alpha_{n}}\right\} M .
$$

Then relation (3.24) is rewritten as

$$
\left\|x_{n+1}-z_{n}\right\|^{2} \leq\left(1-\delta_{n}\right)\left\|x_{n}-z_{n-1}\right\|^{2}+\delta_{n} \theta_{n}
$$

By conditions (i), (ii) and (iii), it is easily found that

$$
\lim _{n \rightarrow \infty} \delta_{n}=0, \quad \sum_{n=1}^{\infty} \delta_{n}=\infty, \quad \lim _{n \rightarrow \infty} \theta_{n}=0 .
$$

We can therefore apply Lemma 2.3 to (3.25) and conclude that $\left\|x_{n+1}-z_{n}\right\|^{2} \rightarrow 0$ as $n \rightarrow \infty$. This completes the proof.

Remark 3.3 Choose the sequences $\left(\alpha_{n}\right)$ and $\left(\beta_{n}\right)$ such that

$$
\alpha_{n}=\frac{1}{(n+1)^{a}} \quad \text { and } \quad \beta_{n}=\frac{1}{(n+1)^{b}}, \quad n \geq 0
$$


where $0<b<a<2 b<1$. It is clear that conditions (i) and (ii) of Theorem 3.2 are satisfied. To verify condition (iii), we compute

$$
\begin{aligned}
\left|\frac{\alpha_{n} \beta_{n-1}-\alpha_{n-1} \beta_{n}}{\alpha_{n}^{2} \beta_{n-1}}\right| & =\frac{1}{\alpha_{n}}\left|1-\frac{\alpha_{n-1} \beta_{n}}{\alpha_{n} \beta_{n-1}}\right| \\
& =(n+1)^{a}\left|1-\frac{(n+1)^{a-b}}{n^{a-b}}\right| \\
& =(n+1)^{a}\left[\left(1+\frac{1}{n}\right)^{a-b}-1\right] \\
& \approx \frac{a-b}{n}(n+1)^{a} \rightarrow 0 .
\end{aligned}
$$

Therefore, $\left\{\alpha_{n}\right\}$ and $\left\{\beta_{n}\right\}$ satisfy all three conditions (i)-(iii) in Theorem 3.2.

\section{Application}

To show an application of our results, we deal with the following problem.

Problem 4.1 Let $0<x_{0}<1$ and define the sequence $\left\{x_{n}\right\}$ by the recursion

$$
x_{n+1}=\left(1-n^{-1 / 2}-n^{-1 / 3}\right) x_{n}+n^{-1 / 3} \frac{x_{n}^{2}}{1+x_{n}} .
$$

At which value does $\left\{x_{n}\right\}$ approach as $n$ goes to infinity?

We claim that $\lim _{n \rightarrow \infty} x_{n}=0$ and it can be easily derived by applying Theorem 3.2.

Proof In order to apply our result, let $H=\mathbb{R}, C=[0,1]$ and define $T: C \rightarrow C$ by

$$
T x:=\frac{x^{2}}{1+x} .
$$

Observe that $T$ is Lipschitzian, pseudocontractive and that $\operatorname{Fix}(T)=\{0\}$. Moreover, if we set $\alpha_{n}=n^{-1 / 2}$ and $\beta_{n}=n^{-1 / 3}$, then

(i) $\lim _{n \rightarrow \infty} \alpha_{n}=0$ and $\sum_{n=0}^{\infty} \alpha_{n}=\infty$;

(ii) $\lim _{n \rightarrow \infty} \frac{\alpha_{n}}{\beta_{n}}=\lim _{n \rightarrow \infty} \frac{\beta_{n}^{2}}{\alpha_{n}}=0$;

(iii) $\lim _{n \rightarrow \infty} \frac{\alpha_{n} \beta_{n-1}-\alpha_{n-1} \beta_{n}}{\alpha_{n}^{2} \beta_{n-1}}=0$.

Then Theorem 3.2 ensures that

$$
\lim _{n \rightarrow \infty} x_{n}=0
$$

Competing interests

The authors declare that they have no competing interests.

\section{Authors' contributions}

All authors contributed equally and significantly in writing this paper. All authors read and approved the final manuscript.

\section{Author details}

'Department of Mathematics, Tianjin Polytechnic University, Tianjin, 300387, China. ${ }^{2}$ Dipartimento di Matematica, Universitá della Calabria, Arcavacata di Rende (CS), 87036, Italy. ${ }^{3}$ Department of Applied Mathematics, National Sun Yat-Sen University, Kaohsiung, 80424, Taiwan. ${ }^{4}$ Department of Information Management, Cheng Shiu University, Kaohsiung, 833, Taiwan. ${ }^{5}$ Center for General Education, Kaohsiung Medical University, Kaohsiung, 807, Taiwan. 


\section{Acknowledgements}

Yonghong Yao was supported in part by NSFC 71161001-G0105. Yeong-Cheng Liou was supported in part by NSC 101-2628-E-230-001-MY3 and NSC 101-2622-E-230-005-CC3.

Received: 15 February 2014 Accepted: 9 May 2014 Published: 23 May 2014

\section{References}

1. Mann, WR: Mean value methods in iteration. Proc. Am. Math. Soc. 4, 506-510 (1953)

2. Geobel, K, Kirk, WA: Topics in Metric Fixed Point Theory. Cambridge Studies in Advanced Mathematics, vol. 28. Cambridge University Press, Cambridge (1990)

3. Goebel, K, Reich, S: Uniform Convexity, Hyperbolic Geometry and Nonexpansive Mappings. Dekker, New York (1984)

4. Reich, S: Weak convergence theorems for nonexpansive mappings in Banach spaces. J. Math. Anal. Appl. 67, 274-276 (1979)

5. Reich, S, Zaslavski, AJ: Convergence of Krasnoselskii-Mann iterations of nonexpansive operators. Math. Comput. Model. 32, 1423-1431 (2000)

6. Suzuki, T: Strong convergence of approximated sequences for nonexpansive mappings in Banach spaces. Proc. Am. Math. Soc. 135, 99-106 (2007)

7. Xu, HK: A variable Krasnoselskii-Mann algorithm and the multiple-set split feasibility problem. Inverse Probl. 22, 2021-2034 (2006)

8. Bauschke, HH, Matoušková, E, Reich, S: Projection and proximal point methods: convergence results and counterexamples. Nonlinear Anal., Theory Methods Appl. 56, 715-738 (2004)

9. Browder, FE, Petryshyn, WV: Construction of fixed points of nonlinear mappings in Hilbert spaces. J. Math. Anal. Appl. 20, 197-228 (1967)

10. Marino, G, Xu, HK: Weak and strong convergence theorems for strict pseudo-contractions in Hilbert spaces. J. Math. Anal. Appl. 329, 336-346 (2007)

11. Chidume, CE, Mutangadura, SA: An example on the Mann iteration method for Lipschitz pseudo-contractions. Proc. Am. Math. Soc. 129, 2359-2363 (2001)

12. Ceng, LC, Petrusel, A, Yao, JC: Strong convergence of modified implicit iterative algorithms with perturbed mappings for continuous pseudocontractive mappings. Appl. Math. Comput. 209, 162-176 (2009)

13. Chidume, $C E$, Abbas, $M$, Ali, B: Convergence of the Mann iteration algorithm for a class of pseudocontractive mappings. Appl. Math. Comput. 194, 1-6 (2007)

14. Chidume, CE, Zegeye, $\mathrm{H}$ : Iterative solution of nonlinear equations of accretive and pseudocontractive types. J. Math Anal. Appl. 282, 756-765 (2003)

15. Ciric, L, Rafiq, A, Cakic, N, Ume, JS: Implicit Mann fixed point iterations for pseudo-contractive mappings. Appl. Math. Lett. 22, 581-584 (2009)

16. Huang, NJ, Bai, MR: A perturbed iterative procedure for multivalued pseudo-contractive mappings and multivalued accretive mappings in Banach spaces. Comput. Math. Appl. 37, 7-15 (1999)

17. Ishikawa, S: Fixed points by a new iteration method. Proc. Am. Math. Soc. 44, 147-150 (1974)

18. Lan, KQ, Wu, JH: Convergence of approximants for demicontinuous pseudo-contractive maps in Hilbert spaces. Nonlinear Anal. 49, 737-746 (2002)

19. Lopez Acedo, G, Xu, HK: Iterative methods for strict pseudo-contractions in Hilbert spaces. Nonlinear Anal. 67, 2258-2271 (2007)

20. Moore, C, Nnoli, BVC: Strong convergence of averaged approximants for Lipschitz pseudocontractive maps. J. Math. Anal. Appl. 260, 269-278 (2001)

21. Qin, X, Cho, YJ, Kang, SM, Zhou, H: Convergence theorems of common fixed points for a family of Lipschitz quasi-pseudocontractions. Nonlinear Anal. 71, 685-690 (2009)

22. Udomene, A: Path convergence, approximation of fixed points and variational solutions of Lipschitz pseudo-contractions in Banach spaces. Nonlinear Anal. 67, 2403-2414 (2007)

23. Yao, Y, Liou, YC, Marino, G: A hybrid algorithm for pseudo-contractive mappings. Nonlinear Anal. 71, 4997-5002 (2009)

24. Yao, Y, Liou, YC, Marino, G: Strong convergence of two iterative algorithms for nonexpansive mappings in Hilbert spaces. Fixed Point Theory Appl. (2009). doi:10.1155/2009/279058

25. Zegeye, $\mathrm{H}$, Shahzad, N, Mekonen, T: Viscosity approximation methods for pseudocontractive mappings in Banach spaces. Appl. Math. Comput. 185, 538-546 (2007)

26. Zhang, $Q$, Cheng, $C:$ Strong convergence theorem for a family of Lipschitz pseudocontractive mappings in a Hilbert space. Math. Comput. Model. 48, 480-485 (2008)

27. Cui, YL, Liu, X: Notes on Browder's and Halpern's methods for nonexpansive maps. Fixed Point Theory 10(1), 89-98 (2009)

28. Yao, $Y, C h e n, R, X u, H K$ : Schemes for finding minimum-norm solutions of variational inequalities. Nonlinear Anal. 72, $3447-3456(2010)$

29. Yao, $Y, X u, H K$ : Iterative methods for finding minimum-norm fixed points of nonexpansive mappings with applications. Optimization 60, 645-658 (2011)

30. Xu, HK: Viscosity approximation methods for nonexpansive mappings. J. Math. Anal. Appl. 298, 279-291 (2004)

31. Yao, Y, Yao, JC: On modified iterative method for nonexpansive mappings and monotone mappings. Appl. Math. Comput. 186, 1551-1558 (2007)

32. Browder, FE: Semicontraction and semiaccretive nonlinear mappings in Banach spaces. Bull. Am. Math. Soc. 74 660-665 (1968)

33. $L u, X, X u, H K$, Yin, $X:$ Hybrid methods for a class of monotone variational inequalities. Nonlinear Anal. 71, 1032-1041 (2009)

34. Zhou, $\mathrm{H}$ : Strong convergence of an explicit iterative algorithm for continuous pseudo-contractions in Banach spaces Nonlinear Anal. 70, 4039-4046 (2009) 
10.1186/1029-242X-2014-206

Cite this article as: Yao et al.: Construction of minimum-norm fixed points of pseudocontractions in Hilbert spaces. Journal of Inequalities and Applications 2014, 2014:206

Submit your manuscript to a SpringerOpen ${ }^{\circ}$ journal and benefit from:

- Convenient online submission

- Rigorous peer review

- Immediate publication on acceptance

Open access: articles freely available online

- High visibility within the field

- Retaining the copyright to your article

Submit your next manuscript at $\gg$ springeropen.com 